\title{
Ensemble coding of memory strength in recognition tests
}

\author{
Chad Dubé $^{1} \cdot$ Ke Tong ${ }^{1} \cdot$ Holly Westfall ${ }^{2} \cdot$ Emily Bauer $^{1}$
}

Published online: 27 March 2019

(C) The Psychonomic Society, Inc. 2019

\begin{abstract}
Recent work by Benjamin and colleagues (Psychological Review, 116 (1), 84-115, 2009; Journal of Experimental Psychology: Learning, Memory, and Cognition, 39(5), 1601-1608, 2013) suggests that recognition memory decisions are corrupted by random variability in decision criteria. This conclusion, which explains several anomalies in the recognition literature, was based on fits of the Noisy Decision Theory of Signal Detection (NDT) to a novel task: ensemble recognition. In the ensemble task, participants make Old/New decisions to ensembles of items rather than single items. The NDT assumption that criteria are fixed across ensembles was criticized by Kellen, Klauer, and Singmann (Psychological Review, 119 (3), 457-479, 2012), and defended by Benjamin (Psychological Review, 120, 720-726, 2013). Little attention, however, has been paid to the assumption of the bestfitting NDT model that participants solve the ensemble task by calculating the average memory strength of items in the probe display. We review evidence of summary statistical representation in visual perception and short-term memory that suggests the aggregation hypothesis is plausible, and hold it up to test in three experiments using the direct ratings procedure. Although we conclude that participants can produce estimates of average probe memory strength at test, in line with the assumptions of NDT, the mechanisms and strategies used to produce such estimates remain unclear.
\end{abstract}

Keywords Recognition memory $\cdot$ Visual perception $\cdot$ Signal detection $\cdot$ Ensemble $\cdot$ Summary statistical representation

Modern accounts of recognition memory often involve a core derived from Signal Detection Theory (SDT; Egan, 1958; MacMillan \& Creelman, 2005). As illustrated in Fig. 1, SDT assumes that recently-encountered items (Old, aka Targets) and items last encountered outside the lab (New, aka Lures) have memory strength values that are normally distributed with (possibly) unequal variance. On an item recognition test, the strength of a given test probe is compared to a criterion value of strength, and if the probe strength exceeds the

Chad Dubé

chaddube@usf.edu

Ke Tong

ketong@mail.usf.edu

Holly Westfall

hwestfal@uci.edu

Emily Bauer

ebauer@mail.usf.edu

1 Department of Psychology, University of South Florida, PCD 4118G, 4202 East Fowler Avenue, Tampa, FL 33620-7200, USA

2 Department of Cognitive Sciences, University of California Irvine, 3151 Social Sciences Plaza, Irvine, CA 92697-5100, USA criterion, the participant responds "Old," and if it does not he/she responds "New." The Hit Rate (HR) and False Alarm Rate (FAR) are modeled as integrals of the Target and Lure curves bounded by the criterion and infinity.

Recent years have seen increased evidence that participants' response criteria may not be fixed across trials, contrary to the assumptions of classical SDT. The evidence, reviewed in detail by Benjamin, Diaz, and Wee (2009), frequently revolves around anomalous behavior of the z-transformed receiver operating characteristic, or zROC (see also Balakrishnan \& MacDonald, 2008; Mueller \& Weidemann, 2008). In discussing such anomalies, Benjamin et al. frequently point to parallels in the perception literature, in which SDT saw its first application to human data. For instance, in the memory literature Van Zandt (2000) found that the slope of the zROC increases with the bias to respond "Old" on memory tests. This finding, replicated by Ratcliff and Starns (2009), echoes earlier work in tone detection conducted by Schulman and Greenberg (1970). In that literature, Treisman and Faulkner (1984) explained the finding by assuming systematic variability in the response criterion from one trial to the next. Paralleling these developments in the perception literature, Benjamin et al. (2009) explained the anomaly in the recognition literature by also assuming noise in the decision 


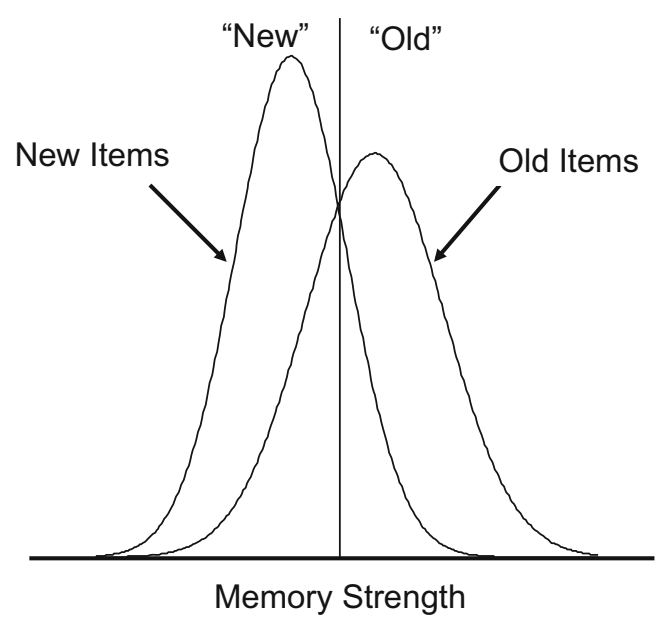

Fig. 1 Unequal-variance signal detection theory (SDT)

criteria (for an alternative but related account see Mueller \& Weidemann, 2008). However, the noise in the NDT model is random rather than a systematic sequential dependency of the sort assumed by Treisman and Faulkner (1984).

In their study, Benjamin et al. also adopted a perceptual task for use in recognition memory: ensemble recognition. Originally used for absolute identification of auditory stimuli by Nosofsky (1983), the ensemble task for recognition involved the presentation of one, two, or four items in a single display, all of which were either Old or New. Participants were asked to make an Old/New judgment to the ensemble. The purpose of using the task was to allow Benjamin et al. to tease apart the contributions of representational and decision variance, the logic being that representational variance alone should increase with ensemble size.

Kellen, Klauer, and Singman (2012) questioned the conclusions reached by Benjamin et al. Their primary concern was the assumption in the NDT modeling that criteria were fixed across ensembles. They concluded that when this assumption is relaxed, evidence for random variability in criteria is greatly reduced. Benjamin (2013) responded to this critique arguing in support of the initial claims.

Interestingly, none of the papers in this series considered the validity of the assumptions made as to how participants solve the ensemble task. Specifically, the NDT model selected by Benjamin et al. and Kellen et al. assumes that participants respond on the basis of a continuous memory strength variable, and average the strengths of the individual items in an ensemble, comparing the result to the response criterion. Are participants actually able to aggregate the memory strength posited by NDT in this way?

Though data from memory tasks are scant, there is a wealth of data relating to aggregation of visual and auditory features in ensemble tasks. Although one must be cautious in generalizing across domains, the degree of overlap between the perception and recognition literatures in terms of the phenomena discussed by Benjamin et al. and the success of perceptual models applied to memory (Green \& Swets, 1966) suggest a consideration of common mechanisms is not only timely but compelled by the data.

Ensemble coding in visual perception and short-term memory Given the reliance on parallels between the perception and memory literatures in Benjamin et al. (2009) in general, and the importation of the ensemble task in particular, it seems useful to consider the large number of findings from the visual perception literature concerning ensemble coding. In brief, this literature suggests that participants can accurately intuit the central tendency of the visual features in a given ensemble (Dubé \& Sekuler, 2015). In one such study, conducted by Ariely (2001), participants viewed displays containing circles differing in diameter. Following this, they were given recognition tests assessing memory for (1) probe circles varying in whether they had been in the memory set (Member Identification) and (2) probe circles varying in whether they matched the average diameter of the study circles (Mean Discrimination). The results showed that, despite chance Member Identification, participants were quite accurate in Mean Discrimination.

Subsequent work established that representation of the mean of a particular feature dimension occurs for numerous visual tasks including multiple object tracking, rapid serial visual presentation, change detection, and Sternberg scanning, among others (see Dubé \& Sekuler, 2015, for review). Such statistical representations have also been demonstrated for variances (Tong, Ji, Chen, \& Fu, 2015), for high-level features such as facial expression (Sweeney, Grabowecki, Paller, \& Suzuki, 2009), and for auditory as well as visual stimuli (McDermott, Schemitsch, \& Simoncelli, 2013). Averaging occurs for items presented simultaneously as well as sequentially (Albrecht \& Scholl, 2010; Corbett \& Oriet, 2011). Statistical representations such as the remembered average of an ensemble's features appear to influence visual shortterm memory (VSTM) retrieval in the absence of any requirement for participants to compute an average, and obligatory averaging's effects appear to be greatest when visual attention is divided at encoding (Dubé, Zhou, Kahana, \& Sekuler, 2014; Dubé \& Sekuler, 2015). A recent study by Oriet and Hozempa (2016) has demonstrated such incidental encoding of statistical information for items presented over time with stimulus durations, feedback intervals, and study-test intervals comparable to those commonly used in studies of categorization (e.g., Nosofsky, 1987), suggesting that representations of central tendency may have a low decay rate and may survive beyond the decay of items in the short-term store, to be transferred to the long-term store.

Do such phenomena extend to the perception of memory strength, the hypothetical variable that is assumed to drive 


\section{Strength Rating at Test}
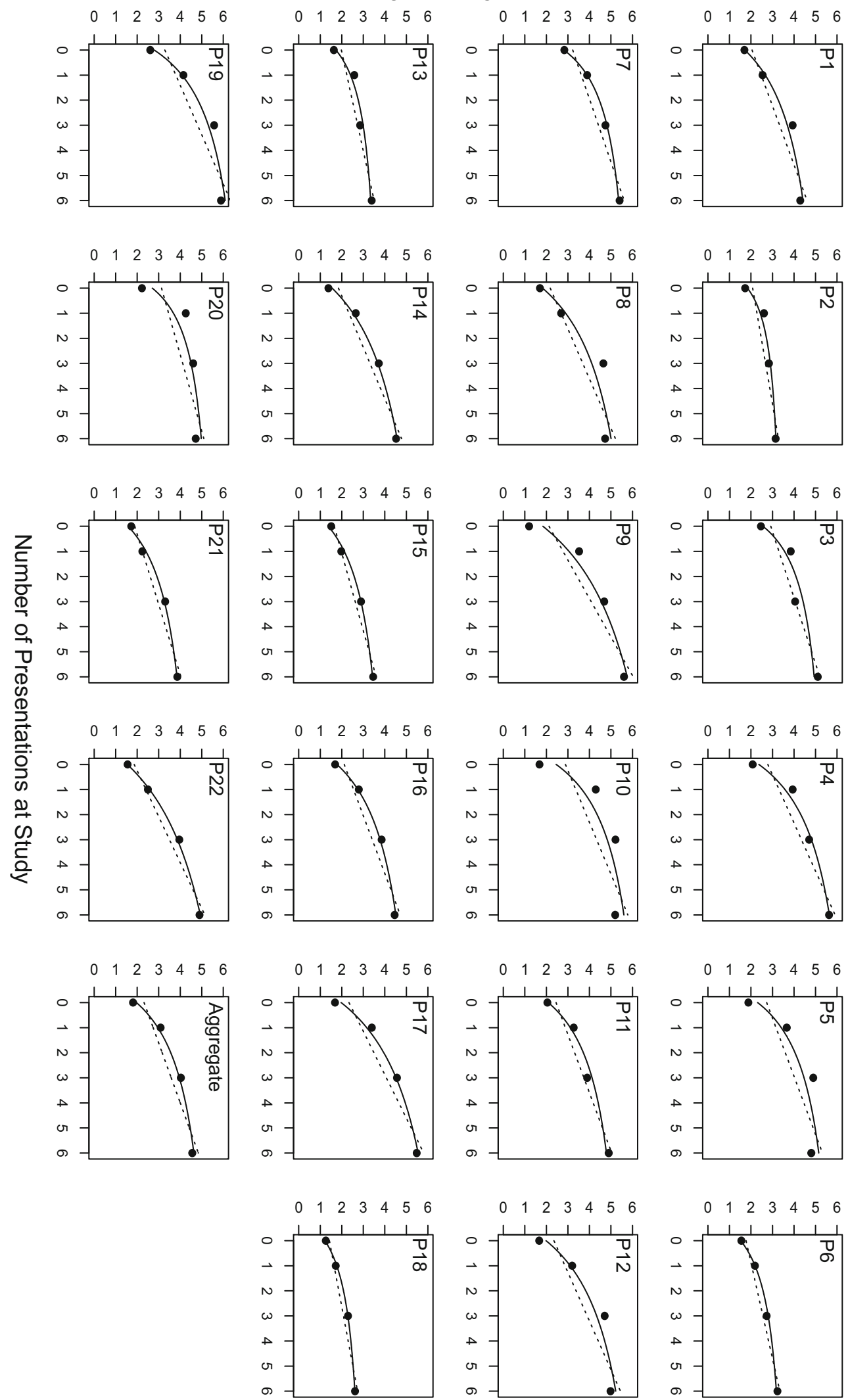

Fig. 2 Ratings of recognition probe memory strength as a function of number of presentations of test probes at encoding. Individual-participant data and aggregate data are displayed, along with fits of the bivariate regression line (dotted) and logistic function (solid) 
Table 1 Values of $\mathrm{R}^{2}$ for the logistic and linear regression fits to the data from Experiment 1

\begin{tabular}{|c|c|c|c|}
\hline Participant & Logistic & Linear & Difference \\
\hline 1 & 0.98 & 0.86 & 0.12 \\
\hline 2 & 0.96 & 0.76 & 0.20 \\
\hline 3 & 0.93 & 0.85 & 0.07 \\
\hline 4 & 0.97 & 0.84 & 0.13 \\
\hline 5 & 0.91 & 0.66 & 0.25 \\
\hline 6 & 0.99 & 0.93 & 0.07 \\
\hline 7 & 1.00 & 0.90 & 0.10 \\
\hline 8 & 0.94 & 0.80 & 0.14 \\
\hline 9 & 0.94 & 0.82 & 0.12 \\
\hline 10 & 0.85 & 0.59 & 0.26 \\
\hline 11 & 0.98 & 0.92 & 0.06 \\
\hline 12 & 0.96 & 0.79 & 0.17 \\
\hline 13 & 0.96 & 0.83 & 0.13 \\
\hline 14 & 0.99 & 0.90 & 0.09 \\
\hline 15 & 0.98 & 0.95 & 0.03 \\
\hline 16 & 1.00 & 0.89 & 0.11 \\
\hline 17 & 0.98 & 0.88 & 0.11 \\
\hline 18 & 1.00 & 0.92 & 0.08 \\
\hline 19 & 0.98 & 0.80 & 0.18 \\
\hline 20 & 0.85 & 0.55 & 0.30 \\
\hline 21 & 0.98 & 0.94 & 0.04 \\
\hline 22 & 1.00 & 0.94 & 0.05 \\
\hline Aggregate & 0.99 & 0.85 & 0.14 \\
\hline
\end{tabular}

Best-fitting functions are in bold

participants' responding in SDT models and NDT? This would seem to imply a model in which, following the initial stages of reading a given item, the retrieval process is engaged and the memory strength output by retrieval (e.g., the activation from a global matching process that retrieves item and context information) is pooled with the outputs from other processed items. At some point prior to the response, the pooled strengths are averaged. The idea that participants can extract the strengths of individual test items during retrieval and subject those strengths to pooling and aggregation, analogously to how the visual system appears capable of pooling and aggregating the visual features of items, seems generally consistent with the pooling and aggregation of familiarity and recollection signals assumed by the continuous dual-process model (Wixted and Mickes, 2010a, b).

Despite the fact that this and related ideas regarding the unitization of paired associates at study (Quamme, Yonelinas, \& Norman, 2007) and the aggregation of probe features in n-item associative recognition probes (Clark \& Shiffrin, 1987) have appeared in the literature, we are unaware of any direct empirical attempt to determine whether participants can aggregate the memory strength of items in a recognition ensemble. Recent work by
Wixted, Vul, Mickes, and Wilson (2017), however, has incorporated this notion in a model of the lineup task. Specifically, out of a number of alternative SDT-based models of sequential and simultaneous lineup identification, the only model that could account for the patterns in estimates of memory strength variance and choice accuracy was one that assumed memory strengths for individual faces were averaged and each face's memory strength was compared to that ensemble memory strength representation.

To determine whether, as assumed by NDT, it is possible for participants to aggregate recognition memory strength, we begin by establishing a set of norms for what the perceived memory strength of an item with a given number of study presentations should be (Experiment 1). We then use this information to derive predictions for what participants' ratings of average strengths across pairs of items should be (Experiment 2). Following this, we report an additional experiment (Experiment 3) designed to test an alternative explanation of our results. To preview, we find the results of Experiments 1-3 support the idea that participants can aggregate recognition memory strength. We conclude by discussing the limitations of our investigation and several open questions related to NDT's aggregation assumption, in the General discussion.

\section{Experiment 1: Direct strength ratings of single items}

\section{Participants}

Twenty-two undergraduates at the University of South Florida completed this experiment for course credit. Experiments 1 and 2 were run in parallel to avoid confounding effects of semester, and participants were barred from participating in both experiments. Experiment 3 was run in a separate semester, again with new participants. Our stopping rule for data collection was to stop following accumulation of at least 20 participants' data in each experiment, on the first day available to the first author to begin analysis. All 22 participants were included in the analysis.

\section{Materials and design}

Stimuli were English nouns from Francis and Kučera (1982) with frequencies of 20-50/million. For each participant, two lists of 72 targets and 72 lures were randomly drawn from the pool of 600 words. Word repetition was manipulated within-subjects so that for the 72 study words on each list, 24 were presented once, 24 were presented three times, and 24 were presented six times per list. Thus, in total, counting the single test presentation of each item, the items were presented once (Lures), twice, four times, and seven times. 
Rating of Average Strength at Test
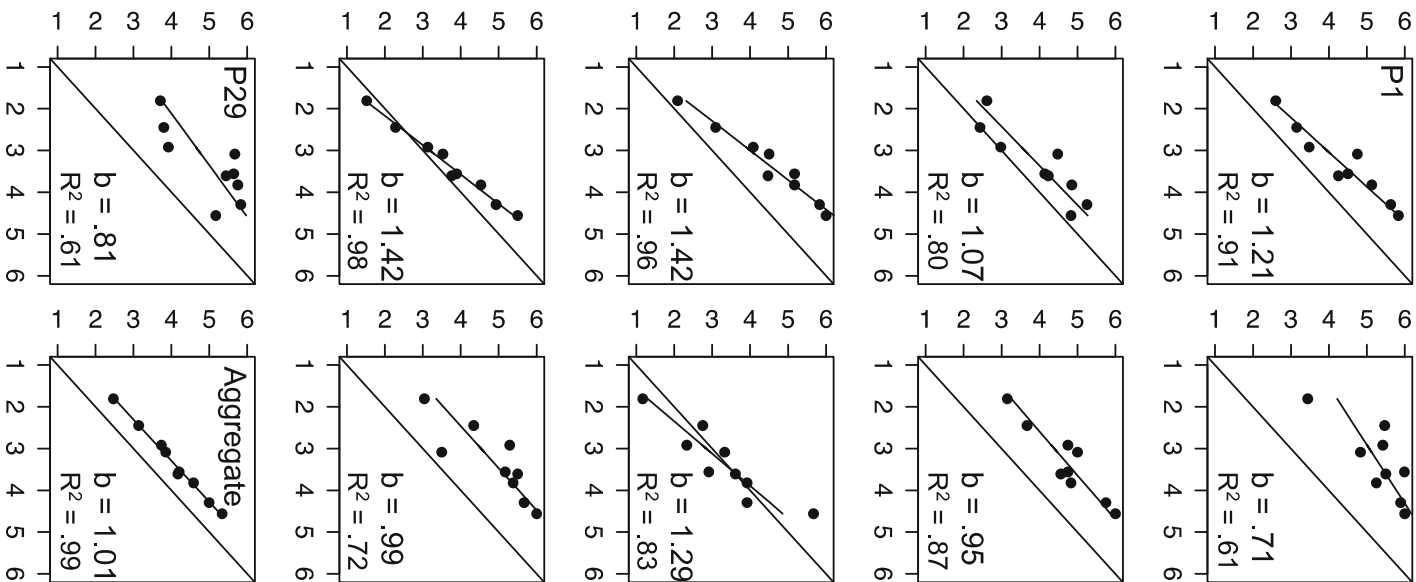

$\begin{array}{llllll}1 & 2 & 3 & 4 & 5 & 6\end{array}$

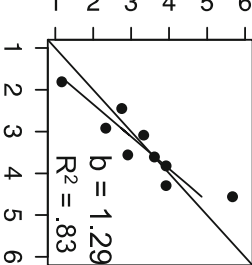

$\begin{array}{llllll}1 & 2 & 3 & 4 & 5 & 6\end{array}$

$\begin{array}{llllll}1 & 2 & 3 & 4 & 5 & 6\end{array}$
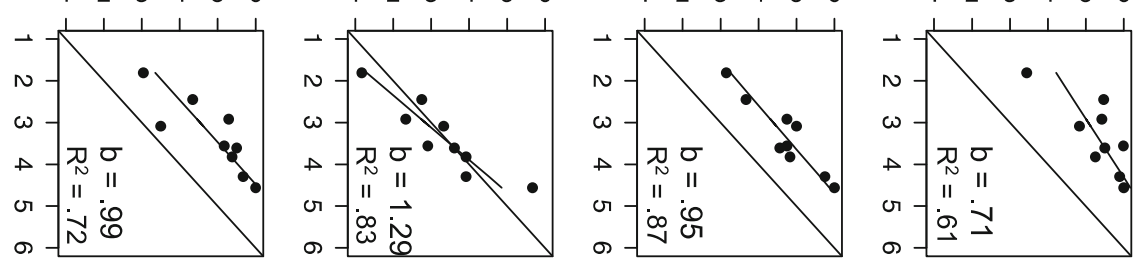

$\begin{array}{llllll}1 & 2 & 3 & 4 & 5 & 6\end{array}$

$\begin{array}{llllll}1 & 2 & 3 & 4 & 5 & 6\end{array}$

$\begin{array}{llllll}1 & 2 & 3 & 4 & 5 & 6\end{array}$

$\begin{array}{llllll}1 & 2 & 3 & 4 & 5 & 6\end{array}$

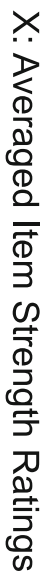
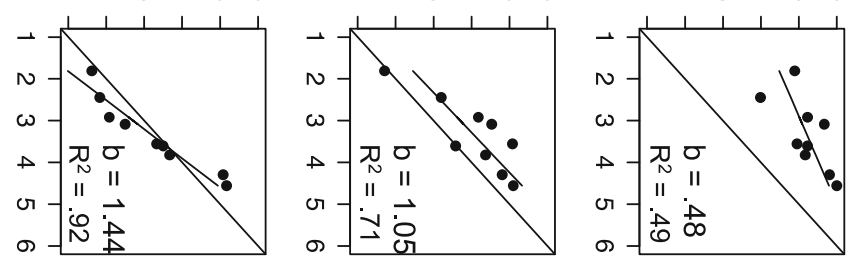

$\begin{array}{llllll}1 & 2 & 3 & 4 & 5 & 6\end{array}$

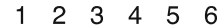

$\begin{array}{llllll}1 & 2 & 3 & 4 & 5 & 6\end{array}$
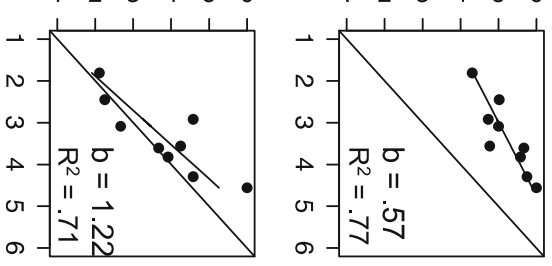

$\begin{array}{llllll}1 & 2 & 3 & 4 & 5 & 6\end{array}$
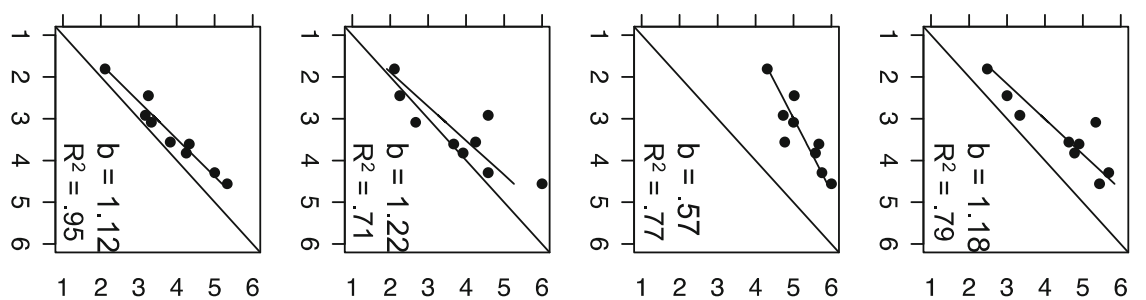

$\begin{array}{llllll}1 & 2 & 3 & 4 & 5 & 6\end{array}$
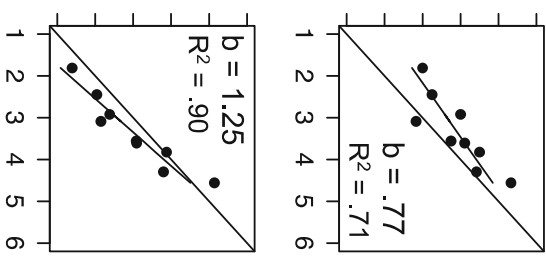

$\begin{array}{lllll}2 & 3 & 4 & 5 & 6\end{array}$
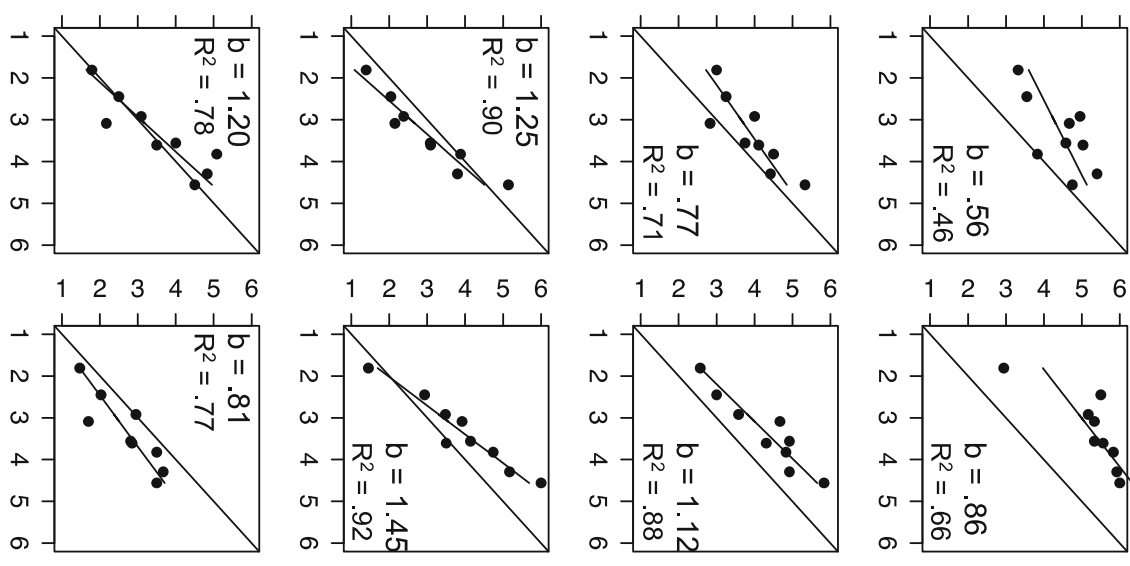

$\begin{array}{llllll}1 & 2 & 3 & 4 & 5 & 6\end{array}$
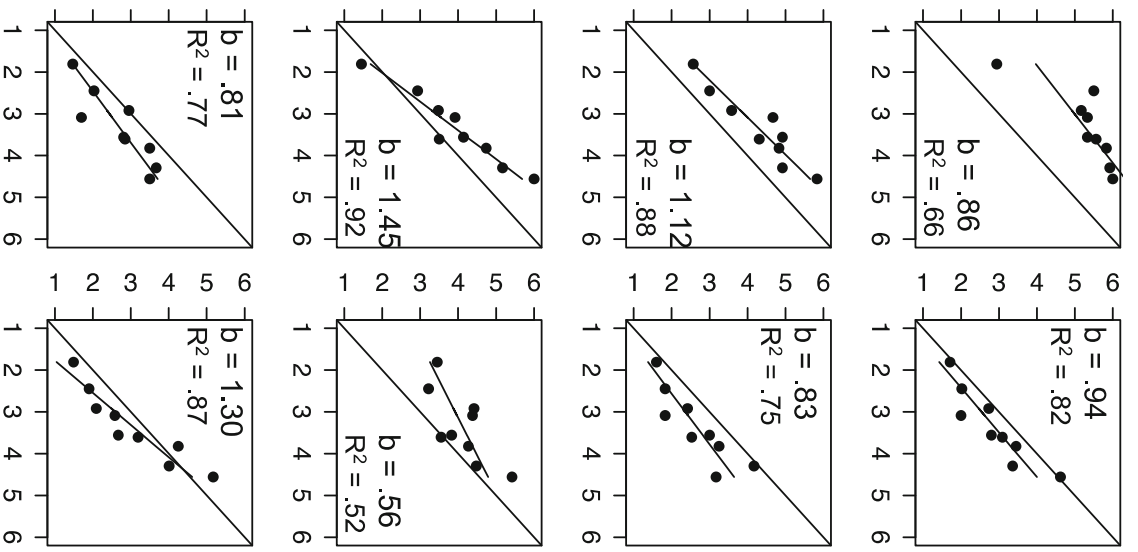

Fig. 3 Ratings of average probe memory strength by participants in Experiment 2 as a function of averaged single-item ratings from particidisplayed, including fits of the bivariate regression line and resulting slope parameters. Unit slope line is included for reference pants in Experiment 1. Individual-participant data and aggregate data are 


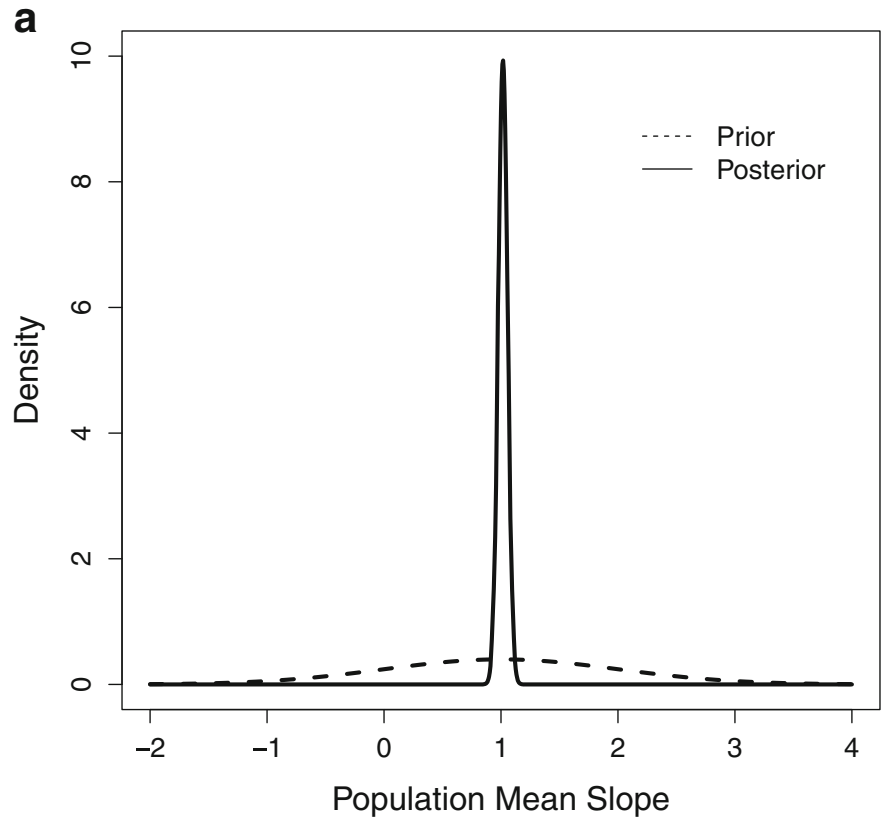

Fig. 4 Hierarchical Bayesian analysis of Experiment 2 results. (A) Prior and posterior over possible values of population mean slope. (B) Posterior median and $95 \%$ credible interval (CrI) (beginning at the 2.5 percentile

\section{Procedure}

The experiment was conducted using desktop PCs in individual booths equipped with 15 -in. LCD monitors with a $1,280 \times 1,024$ resolution. At study, words were presented in white Arial font (height $=.75 \mathrm{~cm}$ ) on a black background. Each study word was presented for $800 \mathrm{~ms}$ with a 200-ms ISI. Word presentation order was fully random, making it possible, though unlikely, to see the same word in succession. Following study, participants completed a 30 -s distractor task involving mental arithmetic.

At test, all 72 targets and 72 lures were centrally presented, one at a time, in a random order. For each, participants indicated the strength of their memory on a scale from 1 (no memory) to 6 (strong memory) (the Direct Ratings task; Mickes, Wixted, \& Wais, 2007). Participants responded by typing a number into a box located below the test word, using up to two decimal places if necessary. Instructions and the test word remained on-screen until the participant responded and pressed the Enter key, prompting the next test word. Two study-test cycles were administered, producing 288 trials in total.

\section{Results}

The results are plotted in Fig. 2. Here it is clear that participants' ratings of their own memory strength increase as a negatively accelerated function of the number of times the test

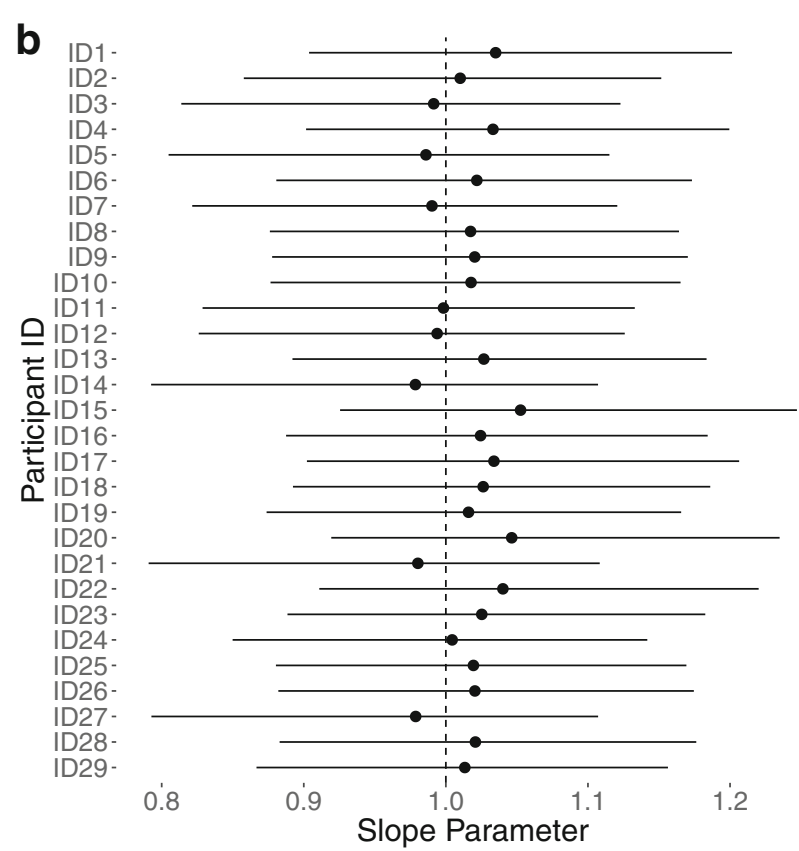

and ending at the 97.5 percentile) for each participant in Experiment 2. For each participant, the value 1 falls within the $95 \% \mathrm{CrI}$

words appeared at study. To verify this intuition, ${ }^{1}$ we fit a scaled logistic function $\left(y=z \frac{x}{x+\theta}\right)$, where number of repetitions is $x, z$ determines the asymptote of the function, and $\theta$ determines the rate of approach. We compared this to the fit of the bivariate linear regression model, both models having two free parameters. In fitting both, we rescaled the horizontal axis by adding a constant (1) to each value, in order to avoid forcing a 0 in the logistic fit. Fits were obtained using the $n l s$ function in $\mathrm{R}$ and the Gauss-Newton algorithm. As is apparent in the figure, the logistic function does an excellent job of describing the data, which appear to be highly consistent across individuals. The linear model, on the other hand, appears to systematically under- and over-predict the data.

Table 1 lists the $R^{2}$ values corresponding to each function fit to each individual, and the group average. The logistic function fits all individuals quite well (smallest $R^{2}=.85$ ). In every case, including all individuals and the group data, the logistic function outperforms the linear model. The average difference in fit is $14 \%$. This substantiates the conclusions drawn from visual inspection: The strength ratings are a negatively accelerated function of number of study presentations,

\footnotetext{
${ }^{1}$ It could be argued the ceiling of 6 in the ratings produces saturation. However, as is clear in Fig. 2, 18/22 (82\%) of the participants produced a rating of 5 or less at the highest repetition value of 6 , with several participants showing asymptotes lower than this. Additionally, Mickes et al. (2011) have shown subjects produce distributions of confidence that cluster at the high end, regardless of the number of points on the scale. This is sensible, given Garner and Hake's (e.g., 1951) work demonstrating that scales with greater than 6 points add little information, amounting to rescaling rather than a change in the distribution of responses.
} 

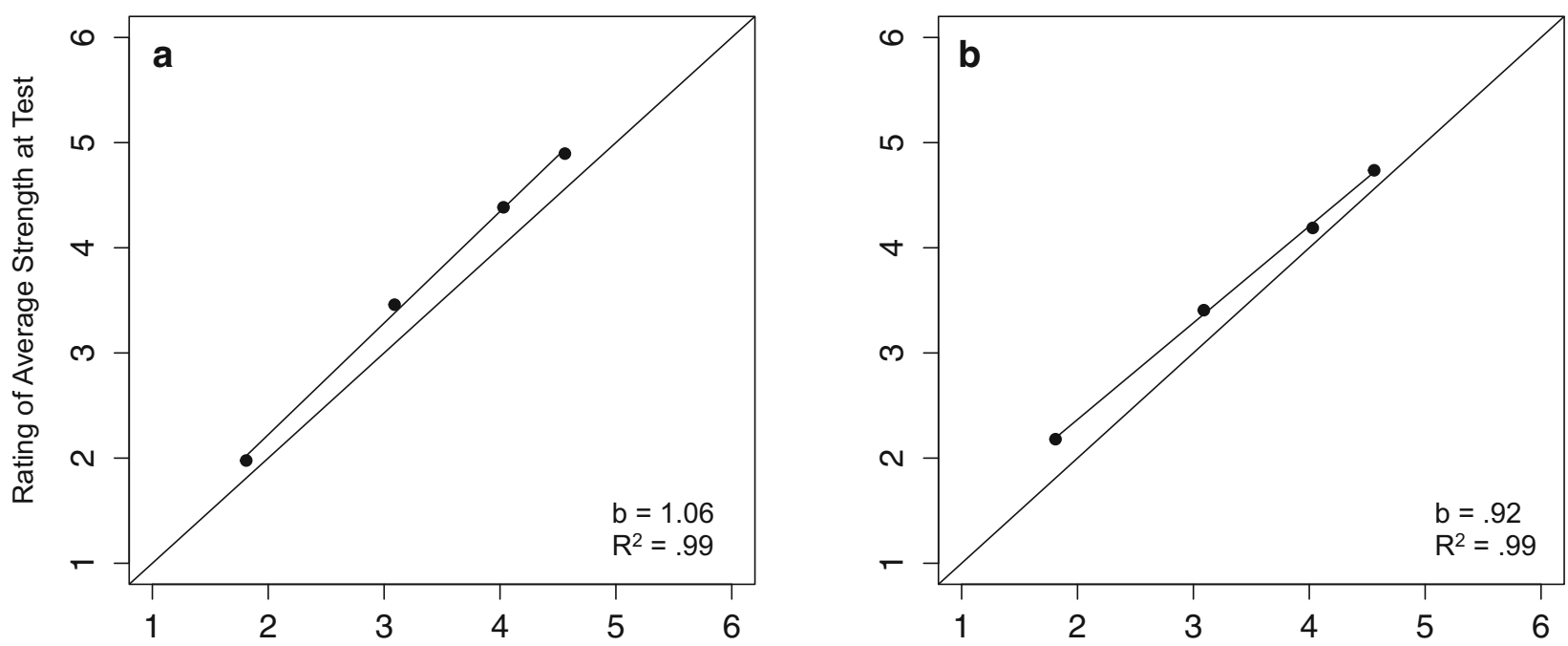

Averaged Item Strength Ratings
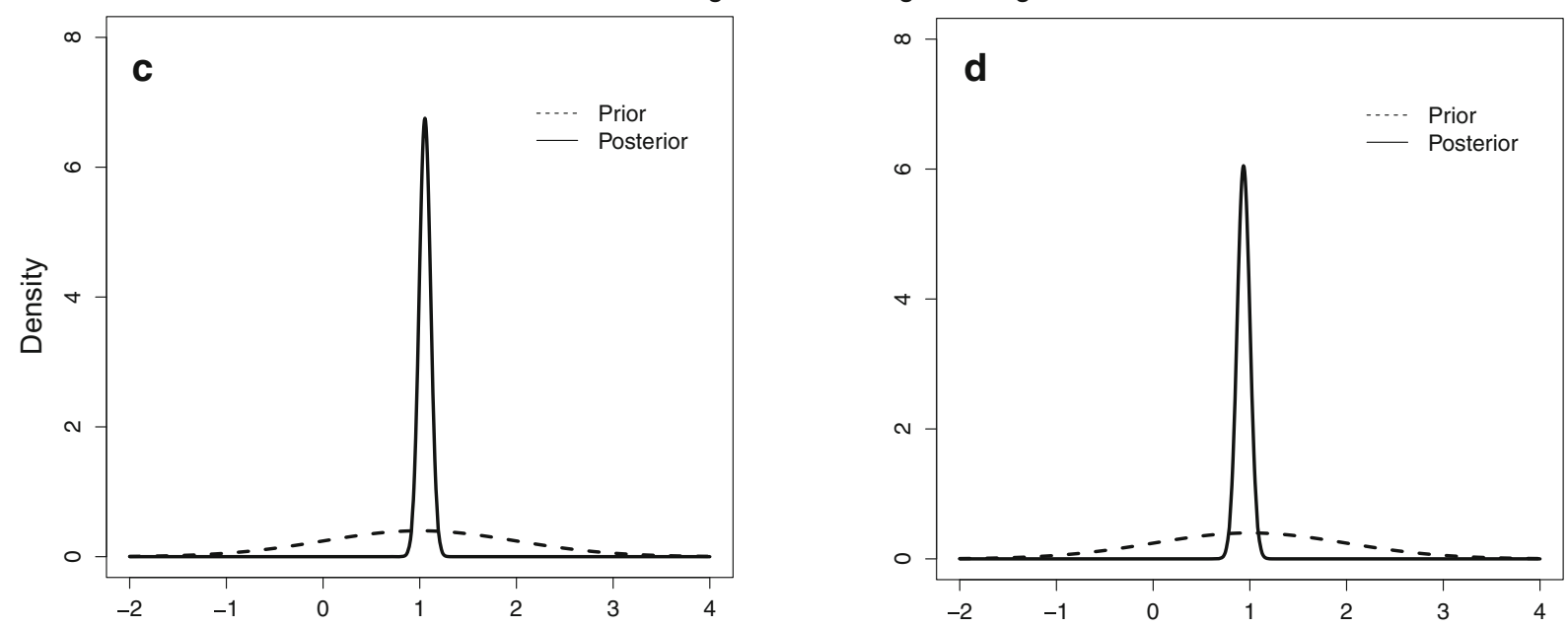

Population Mean Slope
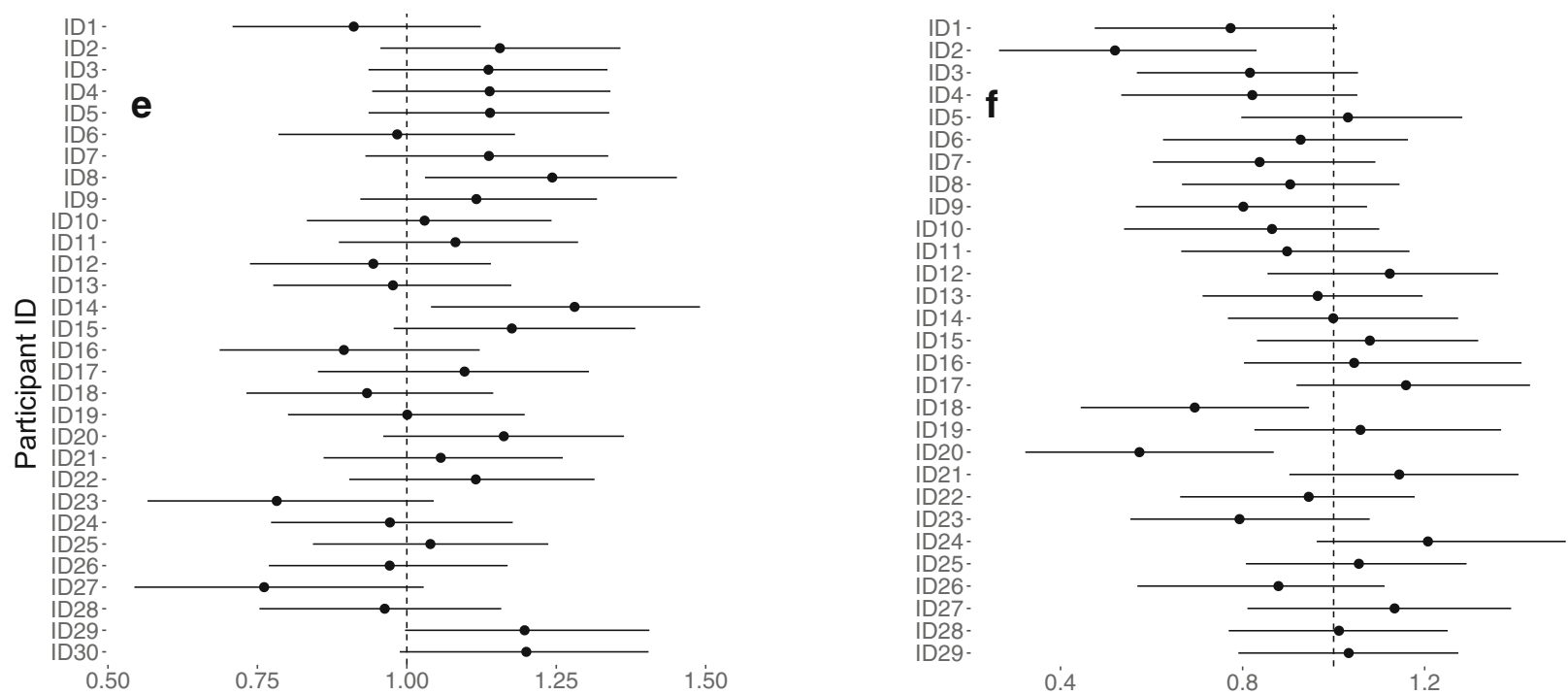

Slope Parameter

Fig. 5 Panels A-B: Regression results for the Summary (A) and Identification (B) groups of Experiment 3. Panels C-D are the corresponding posteriors of the Hierarchical Bayesian analysis of each group. Panels E-F show the 95\% credible intervals (CrIs) for each individual (dots denote medians) 
and this holds for every participant's data. This indicates the Experiment 1 data provide a stable set of norms for memory strength ratings, and a reasonable benchmark for use in the analysis of new participants' data in Experiments 2-3.

An alternative explanation of our results is that participants based their ratings not on strength, but on the number of presentations of the items at study. In other words, that they were implicitly computing Judgments of Frequency (JOFs; Hintzman, 1988). However, zROC slopes derived from JOFs reliably exceed 1 (Hintzman, 2004; Proctor, 1977), unlike those based on confidence ratings, which are typically near .80 (see Benjamin et al., 2009, for review). The slope derived from the direct ratings in this experiment was .78 , and in fits of the unequal-variance SDT model to individual participants' data separately by study repetitions of Targets, the highest value of the SDT zROC slope estimate, $1 / \sigma_{\text {Target }}$, was 1.00 , and this value occurred in only $7 / 66(<11 \%)$ cases (observed and SDT-generated ROCs, along with corresponding values of $\sigma_{\text {Target }}$, can be found in the Appendix). In other words, not a single individual zROC out of 66 cases showed a slope exceeding 1 . These results argue against the JOF interpretation.

Having derived a stable set of strength ratings for items in Experiment 1, we use the group-averages for each repetition

Table 2 Regression parameters and R2 values for Experiment 3, as a function of Condition and predictor variable

\begin{tabular}{|c|c|c|c|c|c|c|c|c|c|}
\hline \multirow[b]{2}{*}{ ID } & \multicolumn{3}{|c|}{ Summary Condition x E1 Norms } & \multicolumn{3}{|c|}{ Identification Condition x E1 Norms } & \multicolumn{3}{|c|}{$\begin{array}{l}\text { Identification Condition x Identification Condition } \\
\text { Individual Predictors }\end{array}$} \\
\hline & b0 & $\mathrm{b}$ & $\mathrm{R} 2$ & b0 & $\mathrm{b}$ & $\mathrm{R} 2$ & b0 & $\mathrm{b}$ & $\mathrm{R} 2$ \\
\hline 1 & 0.15 & 0.84 & 0.97 & 3.12 & -0.07 & 0.16 & 3.18 & -0.19 & 0.08 \\
\hline 2 & -0.25 & -0.25 & 1.31 & 0.99 & 0.29 & 0.41 & 0.88 & -0.19 & 1.09 \\
\hline 3 & 0.22 & 1.16 & 0.99 & 1.18 & 0.61 & 0.82 & -0.05 & 1.09 & 0.93 \\
\hline 4 & -0.60 & 1.38 & 0.95 & 2.37 & 0.36 & 0.84 & 0.38 & 0.93 & 0.64 \\
\hline 5 & 0.70 & 1.05 & 0.91 & -0.30 & 1.25 & 0.98 & -0.10 & 0.99 & 0.99 \\
\hline 6 & 0.87 & 0.77 & 0.95 & 2.65 & 0.41 & 0.82 & 0.23 & 0.93 & 0.97 \\
\hline 7 & 0.96 & 0.98 & 0.93 & 0.02 & 0.91 & 0.99 & 0.18 & 0.97 & 0.99 \\
\hline 8 & -1.24 & 1.69 & 0.92 & 0.55 & 0.88 & 0.97 & 0.32 & 0.89 & 0.99 \\
\hline 9 & -0.76 & 1.38 & 0.93 & -0.53 & 0.98 & 0.91 & 0.22 & 0.95 & 0.99 \\
\hline 10 & -1.24 & 1.18 & 0.92 & 1.92 & 0.52 & 0.90 & 0.15 & 0.95 & 0.99 \\
\hline 11 & -1.15 & 1.43 & 0.98 & -0.62 & 1.15 & 0.99 & -0.33 & 1.04 & 0.99 \\
\hline 12 & 2.04 & 0.41 & 0.66 & 1.29 & 1.02 & 0.91 & -0.04 & 1.01 & 0.99 \\
\hline 13 & 0.82 & 0.77 & 0.81 & 0.83 & 0.85 & 0.86 & 0.17 & 0.92 & 0.97 \\
\hline 14 & 0.58 & 1.29 & 0.80 & -1.06 & 1.39 & 0.97 & 0.05 & 0.98 & 0.99 \\
\hline 15 & -1.52 & 1.67 & 0.95 & 0.53 & 1.13 & 0.97 & 0.44 & 0.85 & 0.94 \\
\hline 16 & -0.98 & 1.11 & 0.92 & -2.35 & 1.88 & 0.98 & 0.31 & 0.95 & 0.98 \\
\hline 17 & 1.85 & 0.66 & 0.97 & -1.00 & 1.60 & 0.98 & -0.06 & 1.00 & 0.99 \\
\hline 18 & -0.26 & 0.98 & 0.99 & 1.25 & 0.43 & 0.73 & 0.16 & 0.92 & 0.99 \\
\hline 19 & 0.87 & 0.79 & 0.96 & -1.79 & 1.65 & 0.99 & 0.01 & 1.00 & 0.99 \\
\hline 20 & -0.42 & 1.37 & 0.97 & 0.28 & 0.48 & 0.78 & 0.14 & 0.93 & 0.98 \\
\hline 21 & -0.97 & 1.35 & 0.87 & -0.76 & 1.52 & 0.95 & 0.00 & 0.99 & 0.99 \\
\hline 22 & 1.77 & 0.73 & 0.96 & 2.13 & 0.56 & 0.99 & 0.63 & 0.86 & 0.99 \\
\hline 23 & -0.36 & 0.78 & 0.89 & -0.54 & 0.87 & 0.89 & -0.16 & 1.11 & 0.99 \\
\hline 24 & -0.53 & 1.11 & 0.99 & -1.77 & 1.85 & 0.90 & -0.05 & 1.01 & 0.99 \\
\hline 25 & 0.33 & 0.99 & 0.98 & 0.76 & 1.00 & 0.98 & 0.34 & 0.92 & 0.99 \\
\hline 26 & 1.17 & 0.67 & 0.80 & 2.77 & 0.32 & 0.81 & 1.68 & 0.57 & 0.95 \\
\hline 27 & 0.03 & 0.65 & 0.76 & 2.84 & 0.66 & 0.98 & -0.16 & 1.01 & 0.99 \\
\hline 28 & 2.54 & 0.31 & 0.74 & 0.51 & 1.02 & 0.96 & 0.41 & 0.92 & 0.99 \\
\hline 29 & -1.84 & 1.78 & 0.97 & 0.73 & 1.01 & 0.98 & -0.67 & 1.12 & 0.99 \\
\hline 30 & 0.25 & 1.25 & 0.99 & & & & & & \\
\hline \multicolumn{10}{|l|}{ Subject } \\
\hline Average & 0.10 & 1.06 & 0.92 & 0.53 & 0.92 & 0.89 & 0.25 & 0.92 & 0.94 \\
\hline Aggregate & 0.10 & 1.06 & 0.99 & 0.53 & 0.92 & 0.99 & 0.17 & 0.96 & 0.99 \\
\hline
\end{tabular}


condition as norms for Experiment 2. In the latter, we use the same encoding design as in Experiment 1. However, at test we ask participants to provide ratings of their average memory strength for pairs of items representing all possible combinations of the number of study repetitions. To test for the ability to average the internal perception of memory strength, we regress these responses onto the averages computed using the norms for items with repetition values corresponding to those of the items in the test pair. If participants can in fact extract average memory strengths at test, the slope of the regression line fit to these data should be close to 1 .

\section{Experiment 2: Ensemble coding of memory strength}

\section{Participants}

Twenty-nine undergraduates at the University of South Florida completed Experiment 2 for course credit. All 29 participants were included in the analysis.

\section{Materials, design, and procedure}

Experiment 2 was identical in materials, design, and procedure to Experiment 1 with the following exceptions: At test, participants were simultaneously presented with two test words and indicated their average memory strength for the pair on a scale from 1 (no memory on average) to 6 (strong memory on average). Test words were paired so that words from each repetition group (including lures) were paired with words from every other repetition group, including selfpairings, and every permutation of repetition group was represented at least three times. Collapsing over order (left or right side of the screen), this produces a total of 10 pairings. A summed study repetition value of 6 , however, can be obtained in two ways: [3,3] and [0,6], hence we collapsed over these two categories in the analysis. ${ }^{2}$ After responding, participants pressed the Enter key to move on to the next test word pair.

\section{Results and discussion}

Results are displayed in Fig. 3, which plots each participant's rating of the average strength of two words as a function of the average of the mean strength ratings of single words presented at rates equal to those of each word in the pair. These singleword rating norms were obtained in Experiment 1. For instance, if a word presented 0 times at study was paired with one presented three times, and the mean item rating for the 0 -

\footnotetext{
$\overline{2}$ Our results were the same regardless of whether we collapsed the ratings in this way. See Appendix Table 4 and Fig. 7.
}

presentation items was 1 in Experiment 1 and for the threepresentation items was 4 , the summed item rating would be 5 . If participants can average the item strengths, then their response should be $.5^{*} 5$ plus a constant noise term, ${ }^{3}$ i.e. $2.5+\varepsilon$.

To assess whether participants were able to form an ensemble representation of memory strength, we fit the bivariate regression line to their data (Fig. 3) and used a Bayesian $t$ to test the hypothesis that the slope of the line, $b$, is 1 . For the Bayesian $t$-test, we used the JASP statistics program (JASP Team, 2016) and adopted the default settings for an uninformed prior distribution: a Cauchy with $r=0.707$. As is clear in Fig. 3, the linear model provided an excellent description of the data (smallest $R^{2}=.46$ ). Bayes Factor produced Odds of 4.94 to 1 favoring the null hypothesis that $b=1$. The aggregate data in Fig. 3 show that the group data are almost exactly as predicted. This fit resulted in the best-fitting regression equation $y=1.01 x+.67$, with $R^{2}=.99$.

A potential issue with the $t$-test we report is that the resulting Bayes Factors do not take into account the uncertainty associated with the individual slope estimates. ${ }^{4}$ For this reason, we also performed a hierarchical Bayesian analysis to test whether the population mean slope, $\mu_{\beta}$, is 1 . We assumed that participants have varying memory ability, that is, some participants perform better or worse at this task independent of any experimental manipulations, and so we included a random effect of participant. We chose a relatively uninformative prior for the population mean $\mu_{\alpha}$ of the intercept and population mean $\mu_{\beta}$ of the slope:

$$
\begin{aligned}
& \mu_{\alpha} \sim \operatorname{Gaussian}(0,1), \\
& \mu_{\beta} \sim \operatorname{Gaussian}(1,1) .
\end{aligned}
$$

An uninformative, flat prior (Gelman, Carlin, Stern, \& Ruben, 1995) was chosen for the population precisions $\tau_{\alpha}$, $\tau_{\beta}$, and $\tau_{\varepsilon}$ of the intercept, the slope, and the residual error, respectively:

$\tau_{\alpha}, \tau_{\beta}, \tau_{\varepsilon} \sim \operatorname{Gamma}(0.001,0.001)$.

All hierarchical analyses were performed using the R programming language (R Core Team, 2018) in conjunction with the tidyverse package (Wickham, 2017) for data aggregation and manipulation. All hierarchical analyses were implemented in JAGS (Plummer, 2003) using the rjags package (Plummer, 2013). JAGS uses Markov Chain Monte Carlo (MCMC) simulations to approximate the posterior distributions of the parameters of interest. For each analysis, we drew four chains with 52,000 samples and discarded the first 2,000

\footnotetext{
${ }^{3}$ Because the regression is carried out on data obtained in separate groups of participants, there could be between-participant differences in response bias (an overall tendency to use higher or lower values on the scale regardless of memory strength). The intercept in the equation is meant to account for such factors, which should be constant across increments in paired item strength. ${ }^{4}$ We thank an anonymous reviewer for pointing this out.
} 
as burn-in. Convergence of chains was confirmed visually and through the use of the Gelman-Rubin diagnostic (R-hat; Gelman \& Rubin, 1992), which was close to 1 in all cases.

Figure 4A shows the overall posterior and prior distributions for the population mean slope $\mu_{\beta}$. To calculate a Bayes Factor, we used the Savage-Dickey approximation (Dickey, 1971). Using this method, the Bayes Factor is determined by calculating the ratio of the prior and posterior densities at some point value of interest (in this case, where $\mu_{\beta}=1$ ). We found $\mathrm{BF}=23.18$ in favor of $\mu_{\beta}=1$. The posterior medians and $95 \%$ credible intervals $(\mathrm{CrI})$ are presented in Fig. 4B. The CrI level is the posterior probability that the median falls in that range; it is clear from the figure that the $95 \% \mathrm{CrI}$ includes 1 for each participant.

An alternative explanation of our results is that participants did not average, but summed the item strengths in order to rate them. This could also produce a slope of 1 since the ratings from lowest to highest summed strength would equal the corresponding averaged single-item ratings. However, summation implies that the variance of the ratings in Experiment 2 should exceed that of Experiment 1, since assuming zero covariance between item strengths (the combinations of item repetition levels were determined factorially, with zero correlation), the variance of a sum of two variables is equal to the sum of the variables' variances. Averaging, on the other hand, would predict a lower SD in Experiment 2 than 1 since if every participant averaged without error and SD for single ratings could be taken as representative of the population of such responses, $\sigma_{\text {avg }}=\frac{\sigma_{\text {single }}}{\sqrt{2}}$. To test these predictions, we divided each participant's ratings SD from Experiment 2 by the mean SD of single-item ratings in Experiment 1. The mean of this ratio was .83 , arguing against summation. Bayes Factor confirmed this, strongly disfavoring the hypothesis that ratio $=1$, with $B F=$ $1.64 \mathrm{e}^{6}$ in favor of the alternative that the $\mathrm{SD}$ ratio $<1$.

We also performed a Bayesian hierarchical analysis to test that the population mean standard deviation for Experiment 2 was less than the population mean standard deviation for Experiment 1. We chose an uninformative prior for the population mean standard deviation (Gelman, 2006) for Experiment $1 \mu_{\sigma 1}$ and Experiment $2 \mu_{\sigma 2}$ :

$\mu_{\sigma 1}, \mu_{\sigma 2} \sim$ Half-Gaussian $(0, \sqrt{2})$

Similarly, the population standard deviations of the standard deviation for Experiment $1 \sigma_{\sigma 1}$ and Experiment $2 \sigma_{\sigma 2}$ each were assigned a prior of:

$\sigma_{\sigma 1}, \sigma_{\sigma 2} \sim$ Half-Gaussian $(0, \sqrt{2})$.

We were able to compute directly the posterior probability that the population mean standard deviation of Experiment 2 was less than the population mean standard deviation of
Experiment 1 . We estimated the probability to be approximately 0.997 .

A second possibility is that participants subsampled the pairs, randomly choosing one item upon which to base their "average." If this were true, however, the SD for Experiment 2 would be larger for larger differences in item strengths within pairs. SD for items presented 0 and 0 times at study was 1.62 , for 0 and two times it was 1.76 , for 0 and three it was 1.68 , and for items presented 0 and six times it was 1.67. There is no systematic increase in ratings SD as a function of the absolute within-pair difference across these pairs $(b=.001)$. For this reason, we rule out the subsampling interpretation.

One possibility that is difficult to assess in the present data is that participants performed absolute identification and averaged the results, i.e., they covertly assigned ratings to the items separately and computed the average of the two ratings. Experiment 3 increased the size of the memory probe ensemble to four items, and we compared performance of participants in the standard task (Summary group) to a second group of participants who were explicitly required to use the identification strategy (Identification group). That is, the latter participants were required to give single item ratings to each item in the ensemble, one at a time, followed by the average of their ratings. To the extent that the Identification strategy is viable, the Identification condition's regression data should be similar to the data from the Summary condition.

\section{Experiment 3}

\section{Participants}

Fifty-nine undergraduates at the University of South Florida completed Experiment 3 for course credit. Participants were randomly assigned to two conditions, described below: the Summary group $(n=30)$ and the Identification group $(n=29)$.

\section{Materials, design, and procedure}

For each participant, two lists of 72 targets and 24 lures were randomly drawn from the pool of 600 words. At test, participants were presented with one of four types of ensembles on a given trial: Type 1 ensembles included four lures, Type 2 ensembles included four words that were presented once during study, Type 3 ensembles included four words that were presented three times during study, and Type 4 ensembles included four words that were presented six times during study. Six ensembles of each type were presented during each test list, for a total of 24 ensembles per list; presentation order of the ensembles was randomized.

There were two groups of participants. In the Summary group, participants were asked to indicate how strong their memory was, on average, for the ensemble just as in 
Experiment 2. In the Identification group, participants had two tasks for a given ensemble probe: First, they were presented with the four-word ensemble and entered their memory strength for each individual word, on a scale from 1 (no memory for the word) to 6 (strong memory for the word), by typing each number into a response box that appeared below the ensemble, using up to two decimal places if necessary. Words were cued for response, one at a time, by outlining them with a yellow box. Second, without viewing the test ensemble again, participants were instructed to enter the average of the numbers they had entered for the four words they were just shown. All other details were as in Experiment 2.

\section{Results}

Regression results for the aggregate data are displayed visually in Fig. 5A-B. Parameter values and $R^{2}$ for all individual participants, subject averages, and the aggregate data are included in Table 2. As is clear in Fig. 5A-B and Table 2, the simple linear model again provided an excellent description of the data (mean of participants' $R^{2}=.92$ and .89 for the Summary and Identification groups, respectively). The fit to the aggregate data resulted in the best-fitting regression equation $y=1.06 x+.10$, with $R^{2}=.99$ for the Summary condition and $y=.92 x+.53$, with $R^{2}=.99$ for the Identification condition, and these values are comparable to the corresponding subject averages in Table 2. For the Summary group, Bayes Factor produced Odds of 3.53 to 1 favoring the null hypothesis that $b=1$. For the Identification group, a similar pattern holds, with $\mathrm{BF}_{01}=3.52$. When the Identification participants' estimates of the average of their ratings are regressed against the true averages of their actual ratings (i.e., the predictors of each particular individual, column 3 of Table 2), the slopes relating the two are not credibly different from $1\left(\mathrm{BF}_{01}=1.28\right.$, $95 \% \mathrm{CVI}=[.34,1.05])$ and the fits are consistently at ceiling, with $19 / 30$ participants having an $R^{2}=.99$ and nearly all participants (28/30) with $R^{2}>.90$.

As in Experiment 2, we also performed a hierarchical Bayesian analysis to test that the population mean slope $\mu_{\beta}$ $=1$ for the Summary group. Again, we chose relatively uninformative priors for the population mean intercept $\mu_{\alpha}$ and the population mean slope $\mu_{\beta}$ :

$\mu_{\alpha} \sim \operatorname{Gaussian}(0,1)$,

$\mu_{\beta} \sim \operatorname{Gaussian}(1,1)$.

Flat priors were chosen for the population precisions $\tau_{\alpha}, \tau_{\beta}$, and $\tau_{\varepsilon}$ of the intercept, the slope, and the residual error, respectively:

$\tau_{\alpha}, \tau_{\beta}, \tau_{\varepsilon} \sim \operatorname{Gamma}(0.001,0.001)$.

Figure 5C shows the overall posterior and prior distributions for the Summary group. Using the Savage-Dickey approximation, we found $\mathrm{BF}=11.12$ in favor of $\mu_{\beta}=1$ for the Summary group. Figure 5E shows the individuals' medians and $95 \%$ CrIs. In all but two cases, the $95 \%$ CrI contains 1.

We repeated the hierarchical Bayesian analysis for the Identification group, using the same priors as presented for the Summary group above. The posterior distributions of the slopes for each participant in the Identification Group, including the median and 95\% CrI, are presented in Fig. 5D and F. For the overall prior and posterior distributions for the population mean slope (5D), BF was 9.05 in favor of $\mu_{\beta}=1$. Of the individuals' $95 \%$ CrIs (5F), all but three contain 1 .

Additionally, for the Identification group, we performed a hierarchical Bayesian analysis to test that the population mean slopes relating the participants' estimates of the average of their ratings to the true averages of their ratings were not credibly different from 1 . For this analysis, we used the same priors as listed for the Summary group above. Here, we found $\mathrm{BF}=28.83$ in favor of $\mu_{\beta}=1$ and in all but two cases the $95 \%$ CrIs contained 1 .

As expected, these results support the notion that when participants are asked to indicate their average memory strength, they can do so by using a covert identification strategy in which they assign ratings to the separate items in the ensemble and then average the ratings, since this is what we required participants in the Identification group to do and the two groups produced very similar results. It is thus difficult to tell, on the basis of the results of the current experiments, how participants compute ensemble memory strength estimates. Nonetheless, the results are in line with the NDT assumption that they can indeed derive such estimates.

One possible explanation of our results is that the Identification participants fell back on an implicit JOF strategy in response to the instructions. As in Experiment 1, however, the zROC data show no evidence of slopes exceeding 1 (slopes averaged .85, .83, and .77 for zROCs with Targets presented $1 \times, 3 \times$, and $6 \times$ ). These results are again inconsistent with the JOF interpretation.

\section{General discussion}

The Noisy Decision Theory of Signal Detection (NDT) selected by Benjamin et al. (2009) was the subject of an extensive critique by Kellen et al. (2012). This criticism focused on the effects of parametric restrictions on response criteria as a function of ensemble size, a decision that was later defended by Benjamin (2013). Less focus has been given to the assumption of the model that participants average the strengths of the memory probes presented in the ensemble task in order to determine their responses. A wealth of data from visual psychophysics and short-term memory suggests participants are fairly accurate in their intuitive judgments as to the central tendency of the features of visual items, and such data have 
been obtained for a variety of perception and short-term memory tasks and in modalities other than vision (see Dubé \& Sekuler, 2015 for review).

Given the strong reliance on findings from perception in Benjamin et al. (2009) and the authors' careful illustration of the many parallels between the domains of recognition and perception, it seemed appropriate to ask the question of whether summary statistical representation might extend to the internal perception of memory strength. In line with the NDT model, we found evidence that participants are in fact capable of extracting a statistical representation of memory strength at test.

Though our results are consistent with NDT's assumption that participants operate on the basis of memory strength and are capable of aggregating it, these conclusions are by no means definitive. One potential limitation of the current work is its dependence on an interval scaling assumption, which has long been a subject of controversy in psychophysics (Townsend \& Ashby, 1984; Shepard, 1981; Rouder, Pratte, \& Morey, 2010). In the memory domain, Mickes, Wixted, and Wais (2007) found close agreement between the parameters of direct ratings distributions and estimates of latent strength distribution parameters from fits of the SDT model to zROC data. This was subsequently challenged by Rouder, Pratte, and Morey (2010), who argued the interval scale assumption was not testable in the direct ratings data, and defended by Wixted and Mickes (2010), who argued that the correspondence data of Mickes et al. would be an unlikely result in the absence of interval scaling.

In the present case, a similar argument applies: it is unclear how the present results, which are consistently linear in Experiments 2 and 3 as predicted from the assumption of a linear map, could occur in the absence of interval scaling from an internal response onto an overt response choice. One possible explanation is that the mapping from the internal response to a response choice (i.e., choice of rating) is not a linear map, but that participants use a linear transformation to produce an overt response that differs from some initial, covert choice of responses. An example is the Identification strategy, in which participants covertly choose ratings for each of the test items in an ensemble and then calculate the arithmetic average of those ratings in order to choose their final, overt response. As Experiment 3 makes clear, this scenario cannot be ruled out in the present data and experiments. However, if in fact participants are using this particular strategy it is unclear why the nature of the mapping from internal response onto the initial, covert response choices would be an issue of contention since the overt responses used to test the hypotheses of Experiments 2 and 3 would by definition be related to their item responses via a linear map (though in the present experiments, those item responses are estimated from Experiment 1 norms).

The full range of results, i.e., the nonlinear results of Experiment 1 and linear results of Experiments 2 and 3, while they cannot ensure the interval scaling assumption is correct, also do not clearly negate an interval scale. Considering the nonlinearity present in the Experiment 1 data and the linearity of Experiments 2 and 3, several explanations are possible that preserve the linear map assumption. For instance, one sequence of transformations consistent with the data from Experiment $1-3$ as well as the interval scaling assumption would be a negatively accelerated mapping from input to internal response, and a linear map from the internal response onto a rating. Such an account is highly speculative, of course, and requires the assumption that participants do not engage in multiple transformations on response choice (as in the Identification strategy). Additional alternative explanations involving nonlinearities at different and/or multiple stages seem to be possible as well.

The assumption about what is being averaged, i.e., that participants respond on the basis of a continuouslydistributed strength variable, could also be questioned. Although our data are consistent with continuous strength, we have not constructed any alternative to strength-based models. It is conceivable that a new model could be constructed to account for our data, perhaps incorporating response oscillation over discrete mental states, in which there is no memory strength signal to speak of. As the discrete-state framework is expanded to produce more and more sophisticated accounts of recognition data (Kellen, Erdfelder, Malmberg, Dubé, \& Criss, 2016; Klauer \& Kellen, 2018), such a possibility seems increasingly likely.

What are the implications of the foregoing points for our conclusions? First and foremost, our data are consistent with the strength aggregation assumption of NDT, and our data from Experiment 1 do suggest that at some point there is a nonlinearity introduced in the mapping from stimulus to response. We speculate that an explanation of this nonlinearity may have to incorporate the study context associations necessary to differentiate between strength signals from studied and unstudied probes (Anderson \& Bower, 1972). Though the results are consistent with NDT, the results of Experiment 3 make clear that the nature and quality of the process by which participants produce their averaging responses remain unclear. The present data do not allow us to determine, for instance, whether participants draw on aggregated memory strength output (as suggested by the continuous dual-process model) or covertly assign individual strength ratings to the items and average those covert ratings as part of a metacognitive strategy.

We hope that our results will motivate further research on the quite important questions raised by the aforementioned limitations. In sum, these are questions involving the nature of recognition's decision variable, the sequence of mapping operations involved in the ensemble and item tasks, and the precise nature of the averaging that participants appear to be 
doing when confronted with ensembles of recognition probes. The latter is particularly important because it is difficult to conclusively rule out Identification on the basis of the present data alone, yet such as test is clearly a crucial step in evaluating NDT's claims. We speculate that further work on these interrelated questions will reveal additional important connections between the mechanisms of perception and memory.

\section{Appendix}

Table 3 Best-fitting parameters, number of freely-varying data points (nobs), and parameters necessary to fit them (npar), resulting df, and R2, for SDT fit to individual-participant and aggregate data from Experiment 1

\begin{tabular}{|c|c|c|c|c|c|c|c|c|c|c|c|c|c|c|c|}
\hline ID & $\mu_{1}$ & $\mu_{2}$ & $\mu_{3}$ & $\sigma_{1}$ & $\sigma_{2}$ & $\sigma_{3}$ & $c_{1}$ & $c_{2}$ & $c_{3}$ & $c_{4}$ & $c_{5}$ & $n_{\text {obs }}$ & $n_{\mathrm{par}}$ & $d f$ & $R^{2}$ \\
\hline 1 & 0.53 & 1.99 & 2.36 & 1.81 & 1.49 & 1.68 & 0.03 & 1.09 & 1.75 & 2.10 & 2.84 & 19 & 7.00 & 12.00 & 0.99 \\
\hline 2 & 0.64 & 0.64 & 1.15 & 1.58 & 2.26 & 1.73 & 0.00 & 1.09 & 1.62 & 2.02 & 2.68 & 20 & 9.00 & 11.00 & 0.99 \\
\hline 3 & 0.91 & 1.06 & 2.50 & 1.32 & 1.37 & 1.90 & -0.24 & 0.22 & 0.66 & 1.14 & 1.49 & 19 & 11.00 & 8.00 & 0.99 \\
\hline 4 & 1.19 & 1.77 & 2.36 & 1.57 & 1.41 & 1.00 & 0.18 & 0.75 & 1.00 & 1.10 & 1.14 & 17 & 11.00 & 6.00 & 1.00 \\
\hline 5 & 1.17 & 1.93 & 2.12 & 1.44 & 1.00 & 1.43 & 0.33 & 0.74 & 1.10 & 1.44 & 1.79 & 20 & 11.00 & 9.00 & 0.99 \\
\hline 6 & 0.66 & 1.11 & 1.54 & 1.02 & 1.00 & 1.20 & 0.42 & 1.03 & 1.64 & 2.40 & 4.10 & 17 & 11.00 & 6.00 & 1.00 \\
\hline 7 & 1.00 & 2.06 & 2.96 & 1.32 & 1.45 & 1.17 & -1.30 & -0.04 & 0.92 & 1.73 & 15.34 & 12 & 11.00 & 1.00 & 1.00 \\
\hline 8 & 0.52 & 2.37 & 2.17 & 1.71 & 1.64 & 1.15 & 0.36 & 0.84 & 1.34 & 1.78 & 2.11 & 20 & 11.00 & 9.00 & 1.00 \\
\hline 9 & 1.84 & 3.00 & 3.00 & 3.78 & 1.86 & 1.00 & 1.35 & 1.56 & 1.84 & 2.03 & 2.22 & 17 & 10.00 & 7.00 & 1.00 \\
\hline 10 & 1.67 & 2.25 & 2.74 & 1.08 & 1.00 & 1.51 & 0.51 & 1.06 & 1.20 & 1.44 & 1.77 & 20 & 11.00 & 9.00 & 0.99 \\
\hline 11 & 0.66 & 1.22 & 1.63 & 1.69 & 1.81 & 1.00 & 0.51 & 0.59 & 0.74 & 0.97 & 1.48 & 18 & 10.00 & 8.00 & 0.99 \\
\hline 12 & 1.01 & 2.64 & 2.85 & 1.57 & 2.06 & 1.87 & 0.55 & 0.84 & 1.30 & 1.61 & 2.03 & 19 & 11.00 & 8.00 & 1.00 \\
\hline 13 & 0.65 & 0.86 & 1.27 & 1.32 & 1.38 & 1.82 & 0.46 & 1.01 & 1.39 & 1.89 & 2.48 & 20 & 11.00 & 9.00 & 0.99 \\
\hline 14 & 1.01 & 1.71 & 2.26 & 1.20 & 1.00 & 1.17 & 1.05 & 1.38 & 1.67 & 1.93 & 2.17 & 19 & 11.00 & 8.00 & 1.00 \\
\hline 15 & 0.50 & 1.21 & 1.71 & 1.00 & 1.61 & 2.05 & 0.38 & 1.17 & 1.82 & 2.48 & 3.20 & 18 & 11.00 & 7.00 & 0.99 \\
\hline 16 & 0.76 & 1.52 & 2.08 & 1.15 & 1.70 & 1.67 & 0.57 & 0.80 & 1.26 & 1.60 & 1.81 & 19 & 11.00 & 8.00 & 0.99 \\
\hline 17 & 0.34 & 1.65 & 2.92 & 14.25 & 1.00 & 1.45 & 1.07 & 1.09 & 1.09 & 1.09 & 1.11 & 6 & 11.00 & -5.00 & 1.00 \\
\hline 18 & 0.61 & 0.07 & 1.02 & 1.10 & 2.89 & 1.98 & 0.99 & 1.55 & 2.09 & 2.48 & 2.87 & 19 & 11.00 & 8.00 & 0.99 \\
\hline 19 & 0.95 & 2.94 & 3.00 & 1.47 & 1.81 & 1.26 & 0.41 & 0.46 & 0.47 & 0.49 & 0.49 & 8 & 11.00 & -3.00 & 1.00 \\
\hline 20 & 1.51 & 2.01 & 1.91 & 1.78 & 2.14 & 1.68 & -0.07 & 0.52 & 0.95 & 1.21 & 1.32 & 18 & 10.00 & 8.00 & 0.99 \\
\hline 21 & 0.07 & 1.02 & 1.53 & 1.45 & 1.54 & 1.83 & 0.49 & 1.15 & 1.38 & 1.66 & 2.07 & 19 & 11.00 & 8.00 & 0.99 \\
\hline 22 & 0.49 & 1.62 & 2.38 & 1.55 & 1.27 & 1.32 & 0.72 & 1.02 & 1.43 & 1.63 & 1.81 & 20 & 11.00 & 9.00 & 1.00 \\
\hline Grp & 0.80 & 1.46 & 1.81 & 1.33 & 1.30 & 1.21 & 0.40 & 0.85 & 1.17 & 1.42 & 1.72 & 20 & 11.00 & 9.00 & 1.00 \\
\hline
\end{tabular}

Saturated fits are denoted in bold 
Hit Rate
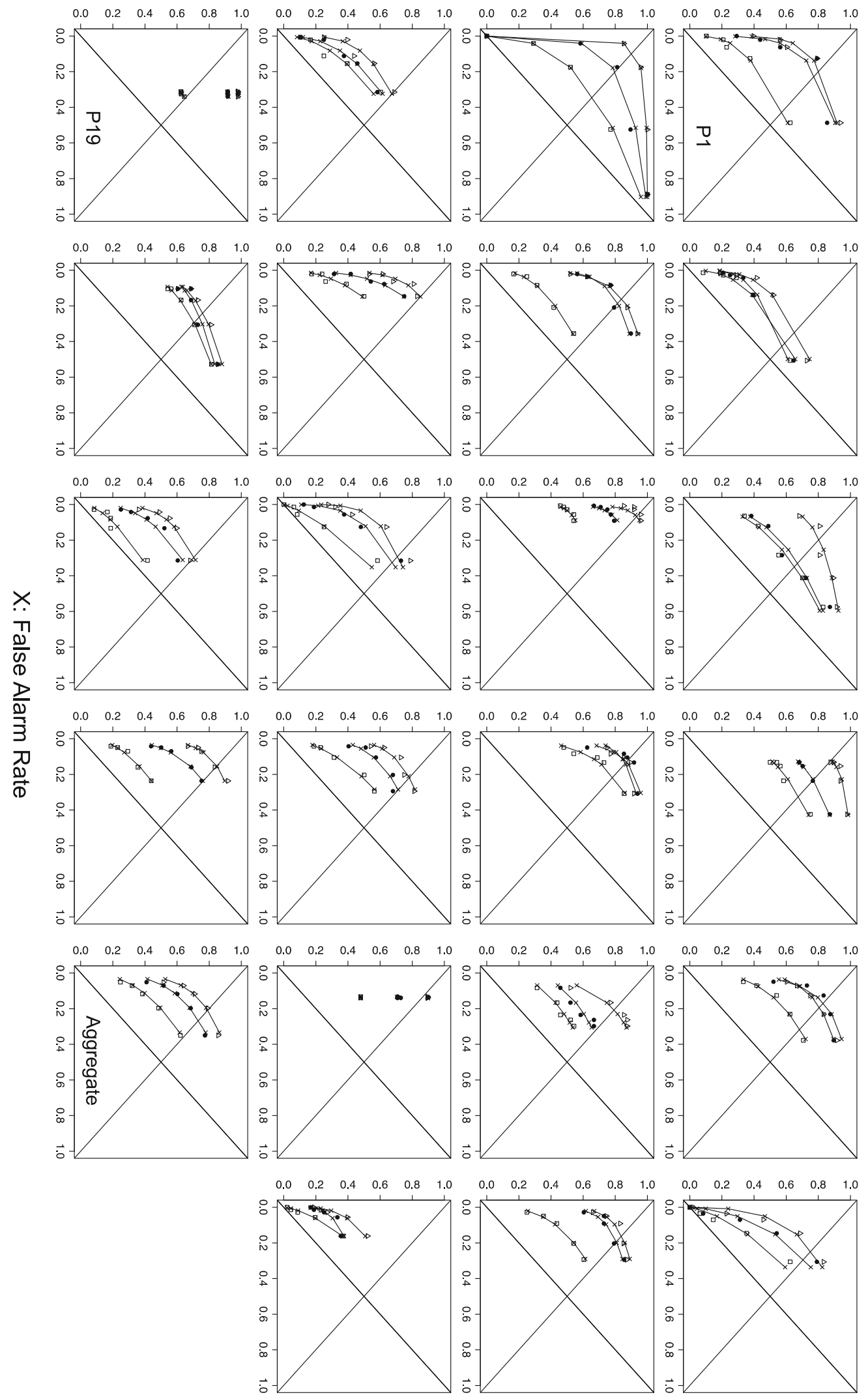

Fig. 6 Observed and SDT-generated ROCs from Experiment 1. Observed data are shown as open squares (1x targets), filled circles

crosses. Included are the data from all 22 individual participants and the $(3 \mathrm{x})$, and open triangles $(6 \mathrm{x})$. Fits from the SDT model are shown as aggregate data. Note: Participants 17 and 19 failed to spread out their use of confidence ratings and the resulting fits are saturated 
Table 4 Slope estimates from fits collapsing over [3, 3] and [0, 6] (Figure 3) and without doing so (Fig. 6) do not differ, $B F_{01}=4.56$

\begin{tabular}{|c|c|c|}
\hline ID & Fig. 3 & Fig. 7 \\
\hline 1 & 1.21 & 1.20 \\
\hline 2 & 0.71 & 0.65 \\
\hline 3 & 0.48 & 0.39 \\
\hline 4 & 1.18 & 1.09 \\
\hline 5 & 0.56 & 0.56 \\
\hline 6 & 0.86 & 0.86 \\
\hline 7 & 0.94 & 0.95 \\
\hline 8 & 1.07 & 1.06 \\
\hline 9 & 0.95 & 0.97 \\
\hline 10 & 1.05 & 1.12 \\
\hline 11 & 0.57 & 0.53 \\
\hline 12 & 0.77 & 0.81 \\
\hline 13 & 1.12 & 1.09 \\
\hline 14 & 0.83 & 0.82 \\
\hline 15 & 1.42 & 1.42 \\
\hline 16 & 1.29 & 1.31 \\
\hline 17 & 1.44 & 1.47 \\
\hline 18 & 1.22 & 1.25 \\
\hline 19 & 1.25 & 1.30 \\
\hline 20 & 1.45 & 1.33 \\
\hline 21 & 0.56 & 0.55 \\
\hline 22 & 1.42 & 1.32 \\
\hline 23 & 0.99 & 1.01 \\
\hline 24 & 0.80 & 0.89 \\
\hline 25 & 1.12 & 1.12 \\
\hline 26 & 1.20 & 1.35 \\
\hline 27 & 0.81 & 0.81 \\
\hline 28 & 1.30 & 1.27 \\
\hline 29 & 0.81 & 0.67 \\
\hline Aggregate & 1.01 & 1.01 \\
\hline
\end{tabular}


Rating of Average Strength at Test
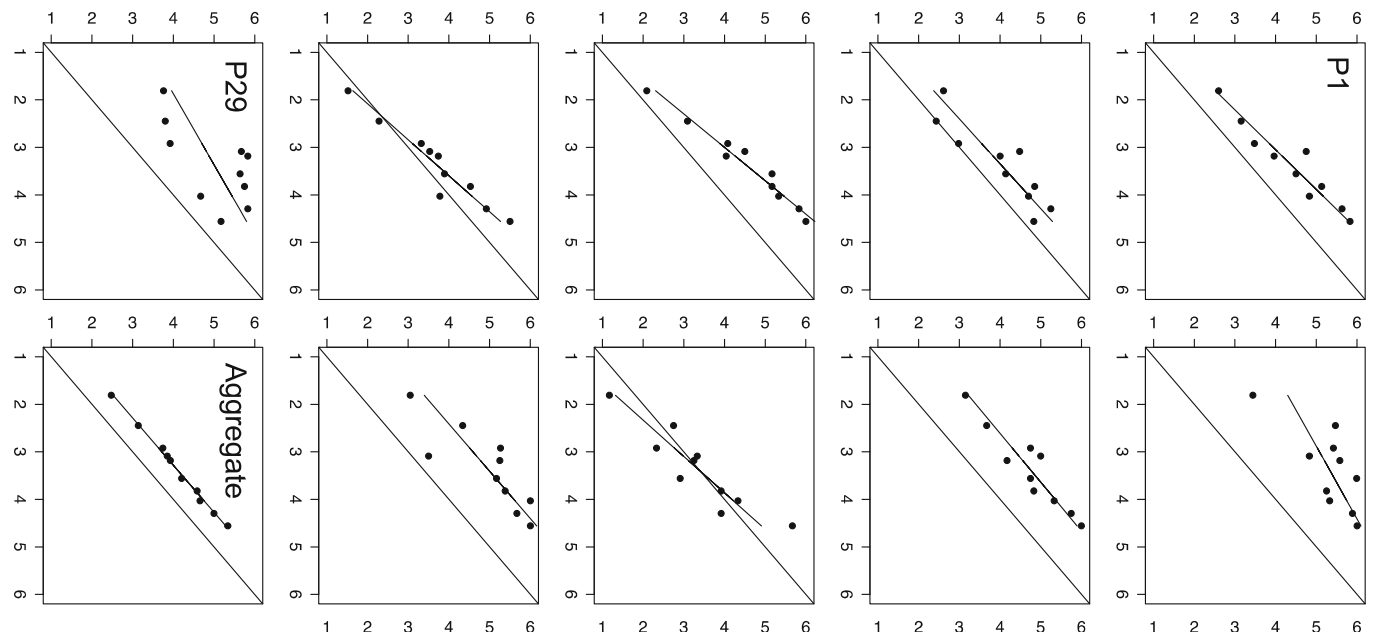

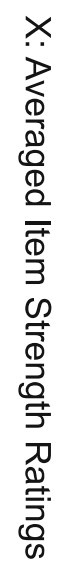
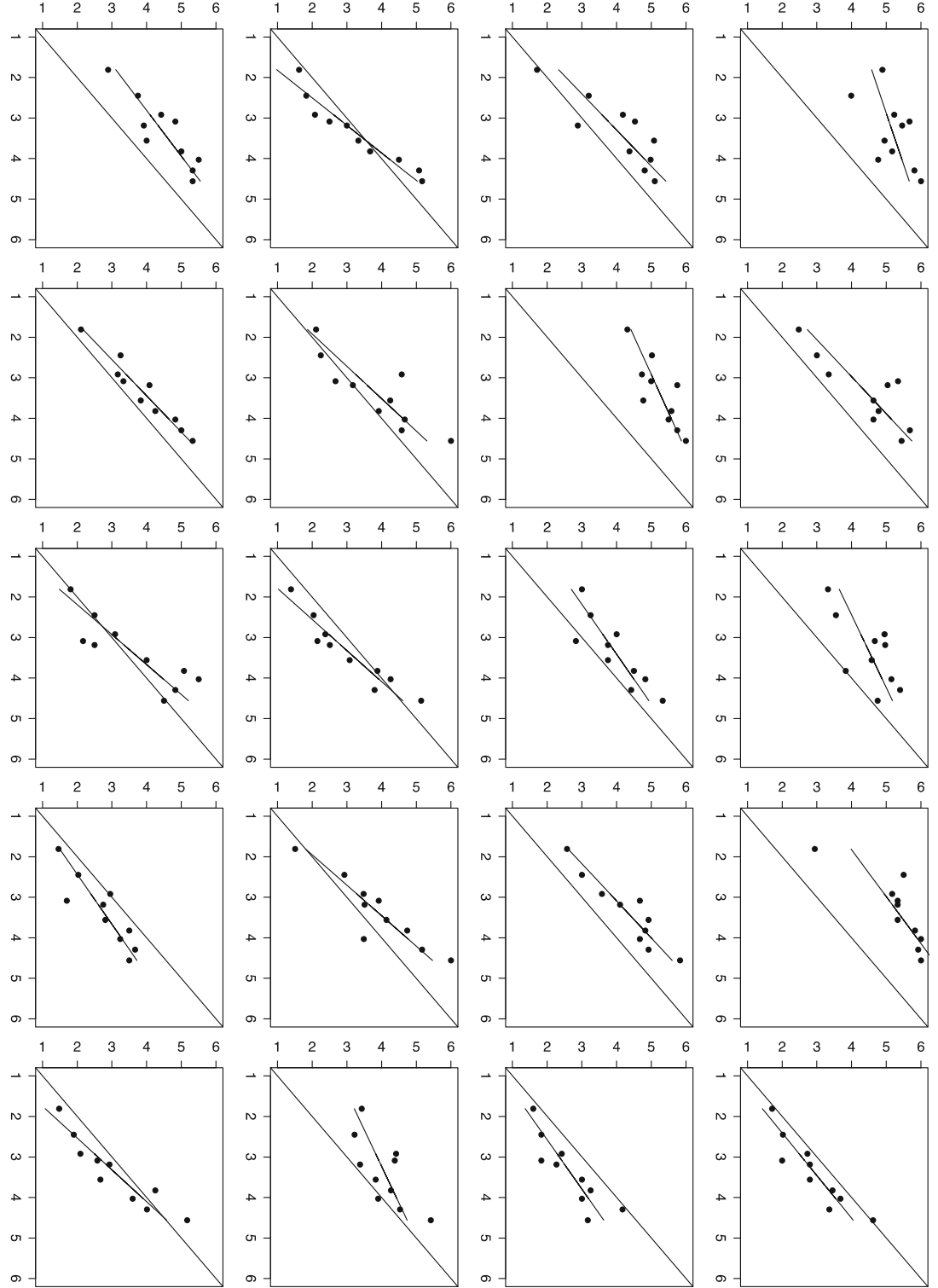

Fig. 7 Ratings of average probe memory strength by participants in Experiment 2 as a function of averaged single-item ratings from particidisplayed, including fits of the bivariate regression line and resulting slope parameters. Unit slope line is included for reference. Data include separate points for $[3,3]$ and $[0,6]$ (see text) 


\section{References}

Albrecht, A. R., \& Scholl, B. J. (2010). Perceptually averaging in a continuous visual world: Extracting statistical summary representations over time. Psychological Science, 21(4), 560567.

Anderson, J. R., \& Bower, G. H. (1972). Recognition and retrieval processes in free recall. Psychological review, 79(2), 97-123.

Ariely, D. (2001). Seeing sets: Representation by statistical properties. Psychological Science, 12(2), 157-162.

Balakrishnan, J. D., \& Macdonald, J. A. (2008). Decision criteria do not shift: Commentary on Mueller and Weidemann (2008). Psychonomic Bulletin \& Review, 15(5), 1022-1030.

Benjamin, A.S. (2013). Where is the criterion noise in recognition? (AlmostS) everyplace you look: Comment on Kellen, Klauer, and Singmann (2012). Psychological Review, 120, $720-726$.

Benjamin, A. S., Diaz, M., \& Wee, S. (2009). Signal detection with criterion noise: Applications to recognition memory. Psychological Review, 116(1), 84-115.

Benjamin, A. S., Tullis, J. G., \& Lee, J. H. (2013). Criterion noise in ratings-based recognition: evidence from the effects of response scale length on recognition accuracy. Journal of Experimental Psychology: Learning, Memory, and Cognition, 39(5), 1601-1608.

Clark, S. E., \& Shiffrin, R. M. (1987). Recognition of multiple-item probes. Memory \& Cognition, 15(5), 367-378.

Corbett, J. E., \& Oriet, C. (2011). The whole is indeed more than the sum of its parts: Perceptual averaging in the absence of individual item representation. Acta Psychologica, 138(2), 289-301.

Dickey, J. M. (1971). The weighted likelihood ratio, linear hypotheses on normal location parameters. The Annals of Mathematical Statistics, 204-223.

Dubé, C., \& Sekuler, R. (2015). Obligatory and adaptive averaging in visual short term memory. Journal of Vision, 15, 1-13.

Dubé, C., Zhou, F., Kahana, M.J., \& Sekuler, R. (2014). Similarity-based distortion of visual short-term memory is perceptual. Vision Research, 96, 8-16.

Egan, J. P. (1958). Recognition memory and the operating characteristic. USAF Operational Applications Laboratory Technical Note.

Francis, W.N., \& Kučera, H. (1982). Frequency analysis of English usage: Lexicon and grammar. Boston: Houghton Mifflin.

Garner, W. R., \& Hake, H. W. (1951). The amount of information in absolute judgments. Psychological Review, 58(6), 446-459.

Gelman, A., \& Rubin, D. B. (1992). Inference from iterative simulation using multiple sequences. Statistical science, 7(4), 457-472.

Gelman, A., Carlin, J. B., Stern, H. S., \& Rubin, D. B. (1995). Bayesian Data Anaylsis, chapter 5. Texts in Statistical Science (pp. 134-141). London: Chapman and Hall.

Gelman, A. (2006). Prior distributions for variance parameters in hierarchical models (comment on article by Browne and Draper). Bayesian Analysis, 1(3), 515-534.

Green, D. M., \& Swets, J. A. (1966). Signal detection theory and psychophysics. New York: Wiley.

Hintzman, D. L. (1988). Judgments of frequency and recognition memory in a multiple-trace memory model. Psychological Review, 95(4), $528-551$.

Hintzman, D. L. (2004). Judgment of frequency versus recognition confidence: Repetition and recursive reminding. Memory \& Cognition, 32(2), 336-350.

Kastner, S., De Weerd, P., Pinsk, M. A., Elizondo, M. I., Desimone, R., \& Ungerleider, L. G. (2001). Modulation of sensory suppression: Implications for receptive field sizes in the human visual cortex. Journal of Neurophysiology, 86(3), $1398-1411$.
Kellen, D., Erdfelder, E., Malmberg, K. J., Dubé, C., \& Criss, A. H. (2016). The ignored alternative: an application of Luce's lowthreshold model to recognition memory. Journal of Mathematical Psychology, 75, 86-95.

Kellen, D., Klauer, K. C., \& Singmann, H. (2012). On the measurement of criterion noise in signal detection theory: The case of recognition memory. Psychological Review, 119(3), $457-479$.

Klauer, K. C., \& Kellen, D. (2018). RT-MPTs: Process models for response-time distributions based on multinomial processing trees with applications to recognition memory. Journal of Mathematical Psychology, 82, 111-130.

Macmillan, N. A., \& Creelman, C. D. (2005). Detection theory: A user's guide. Psychology Press.

McDermott, J. H., Schemitsch, M., \& Simoncelli, E. P. (2013). Summary statistics in auditory perception. Nature neuroscience, 16(4), 493498.

Mickes, L., Wixted, J. T., \& Wais, P. E. (2007). A direct test of the unequal-variance signal detection model of recognition memory. Psychonomic Bulletin \& Review, 14(5), 858-865.

Mickes, L., Hwe, V., Wais, P. E., \& Wixted, J. T. (2011). Strong memories are hard to scale. Journal of Experimental Psychology: General, 140(2), 239

Mueller, S. T., \& Weidemann, C. T. (2008). Decision noise: An explanation for observed violations of signal detection theory. Psychonomic Bulletin \& Review, 15(3), 465-494.

Nosofsky, R. M. (1983). Information integration and the identification of stimulus noise and criterial noise in absolute judgment. Journal of Experimental Psychology: Human Perception and Performance, 9(2), 299-309.

Nosofsky, R. M. (1987). Attention and learning processes in the identification and categorization of integral stimuli. Journal of Experimental Psychology: Learning, Memory, and Cognition, 13(1), 87.

Oriet, C., \& Hozempa, K. (2016). Incidental statistical summary representation over time. Journal of Vision, 16(3), 3-3.

Plummer, M. (2013). rjags: Bayesian graphical models using MCMC. R package version, 3(10).

Proctor, R. W. (1977). The relationship of frequency judgments to recognition: Facilitation of recognition and comparison to recognitionconfidence judgments. Journal of Experimental Psychology: Human Learning and Memory, 3(6), 679-689.

Quamme, J. R., Yonelinas, A. P., \& Norman, K. A. (2007). Effect of unitization on associative recognition in amnesia. Hippocampus, 17(3), 192-200.

R Development Core Team (2008). R: A language and environment for statistical computing. Vienna: R Foundation for Statistical Computing. ISBN 3-900051-07-0, URL http://www.R-project.org.

Ratcliff, R., \& Starns, J. J. (2009). Modeling confidence and response time in recognition memory. Psychological review, 116, 59-83.

Rouder, J. N., Pratte, M. S., \& Morey, R. D. (2010). Latent mnemonic strengths are latent: A comment on Mickes, Wixted, and Wais (2007). Psychonomic Bulletin \& Review, 17(3), 427-435.

Schulman, A. I., \& Greenberg, G. Z. (1970). Operating characteristics and a priori probability of the signal. Attention, Perception, \& Psychophysics, 8, 317-320.

Shepard, R. N. (1981). Psychological relations and psychophysical scales: On the status of "direct" psychophysical measurement. Journal of Mathematical Psychology, 24(1), 21-57.

JASP Team (2016). JASP (Version 0.7.5.5) [Computer software].

Tong, K., Ji, L., Chen, W., \& Fu, X. (2015). Unstable mean context causes sensitivity loss and biased estimation of variability. Journal of vision, 15(4), 15-15.

Townsend, J. T., \& Ashby, F. G. (1984). Measurement scales and statistics: The misconception misconceived. Psychological Bulletin, 96, 394-401. 
Treisman, M., \& Faulkner, A. (1984). The effect of signal probability on the slope of the receiver-operating characteristic given the by rating procedure. British Journal of Mathematical and Statistical Psychology, 37, 199-215.

Van Zandt, T. (2000). ROC curves and confidence judgments in recognition memory. Journal of Experimental Psychology: Learning, Memory, and Cognition, 26, 582-600.

Wickham, H. (2017). The tidyverse. R package ver. 1.1. 1.

Wixted, J. T., \& Mickes, L. (2010a). Useful scientific theories are useful: A reply to Rouder, Pratte, and Morey (2010). Psychonomic Bulletin \& Review, 17(3), 436-442.
Wixted, J. T., \& Mickes, L. (2010b). A continuous dual-process model of remember/know judgments. Psychological Review, 117(4), 10251054.

Wixted, J.T., Vul, E., Mickes, L., \& Wilson, B. (2017). Models of lineup memory. Paper presented at the $58^{\text {th }}$ Annual Meeting of the Psychonomic Society.

Publisher's note Springer Nature remains neutral with regard to jurisdictional claims in published maps and institutional affiliations. 